\title{
MORITA THEORY IN STABLE HOMOTOPY THEORY
}

\author{
BROOKE SHIPLEY
}

\begin{abstract}
We discuss an analogue of Morita theory for ring spectra, a thickening of the category of rings inspired by stable homotopy theory. This follows work by Rickard and Keller on Morita theory for derived categories. We also discuss two results for derived equivalences of DGAs which show they differ from derived equivalences of rings.
\end{abstract}

\section{INTRODUCTION}

Although the usual paradigm in algebraic topology is to translate topological problems into algebraic ones, here we discuss the translation of algebra into topology. Specifically, we discuss an analogue of Morita theory for a thickening of the category of rings inspired by stable homotopy theory. Here rings in the classical sense correspond to ordinary cohomology theories, whereas "rings up to homotopy" correspond to generalized cohomology theories. Although these generalized rings have a considerable history behind them, only recent progress has allowed the wholesale transport of algebraic methods into this domain.

The topological analogue of Morita theory is very similar to the following two algebraic versions. To emphasize this similarity we delay discussion of the technical terminology used in these algebraic statements. We include the third condition below since it is the most familiar criterion for classical Morita equivalences, but we concentrate on the equivalence of the first two conditions in the other contexts.

Theorem 1.1. Two rings $R$ and $R^{\prime}$ are Morita equivalent if the following equivalent conditions hold.

(1) The categories of right modules over $R$ and $R^{\prime}$ are equivalent.

(2) There is a finitely generated projective (strong) generator $M$ in Mod- $R^{\prime}$ such that the endomorphism ring $\operatorname{hom}_{R^{\prime}}(M, M)$ is isomorphic to $R$.

(3) There is an $R-R^{\prime}$ bimodule $N$ such that $-\otimes_{R} N:$ Mod- $R \longrightarrow \operatorname{Mod}-R^{\prime}$ is an equivalence of categories.

Next we state a reformulation from [DS1] of Rickard's characterization of equivalences of derived categories [R1, R2]; see also [Ke1, 9.2].

Theorem 1.2. Two rings $R$ and $R^{\prime}$ are derived equivalent if the following equivalent conditions hold.

(1) The unbounded derived categories of $R$ and $R^{\prime}$ are triangulated equivalent.

(2) There is a compact generator $M$ of $\mathcal{D}\left(R^{\prime}\right)$ such that the graded endomorphism ring in the derived category, $\mathcal{D}\left(R^{\prime}\right)(M, M)_{*}$, is isomorphic to $R$ (concentrated in degree zero).

Date: July 27, 2004; 1991 AMS Math. Subj. Class.: 55U35, 55P42.

Research partially supported by an NSF Grant. 
If $M$ satisfies the conditions in (2), it is called a tilting complex.

For the analogue in stable homotopy theory, one must make the appropriate changes in terminology. The real difference lies in the meaning of "ring" and "equivalence"; we devote a section below to defining each of these terms. In Section 2 we introduce "abelian groups up to homotopy" or spectra and the associated "rings up to homotopy" or ring spectra. Although spectra are the main object of study in stable homotopy theory and have been studied for almost forty years, only recent work has made the definitions of ring spectra easily accessible. In Section 3 we consider a notion of "up to homotopy" equivalences of categories, or Quillen equivalences. Here "homotopy" is determined by defining Quillen model structures on the relevant categories. This extra structure allows one to apply standard techniques of homotopy theory in non-standard settings.

Theorem 1.3. [SS2, DS3] The following two statements are equivalent for ring spectra $R$ and $R^{\prime}$.

(1) The Quillen model categories of $R$-module spectra and $R^{\prime}$-module spectra are Quillen equivalent.

(2) There is a compact generator $M$ in $\operatorname{Ho}\left(R^{\prime}\right.$-modules $)$ such that the derived endomorphism ring spectrum $\overline{\operatorname{Hom}}_{R^{\prime}}(M, M)$ is weakly equivalent to $R$.

The proofs of these three statements basically have the same format. In each situation, one can prove that (1) implies (2) by noting that the image of $R$ under the given equivalence has the properties required of $M$. For (2) implies (1), the conditions on $M$ are exactly what is needed to show that the appropriate analogue of $\operatorname{hom}_{R^{\prime}}(M,-)$ induces the necessary equivalence. For example, in the classical statement one asks that $M$ is a finitely generated projective module to ensure that $\operatorname{hom}_{R^{\prime}}(M,-)$ preserves sums (and is exact). In a triangulated category $\mathcal{T}, M$ is compact if $\mathcal{T}(M,-)$ preserves sums. (For example, in $\mathcal{D}(R)$ a bounded complex of finitely generated projectives is compact [BN] and conversely [Ke1, 5.3], [Ne1].) Also, if $M$ is compact then it is a (weak) generator of $\mathcal{T}$ if it detects trivial objects; that is, an object $X$ of $\mathcal{T}$ is trivial if and only if there are no graded maps from $M$ to $X, \mathcal{T}(M, X)_{*}=0$. (For triangulated categories with infinite coproducts this is shown to be equivalent to the more common definition of a generator in [SS2, 2.2.1].) See [Sch3] for a more detailed survey developing the three theorems above.

The topological analogue of Morita theory has had many applications in stable homotopy theory, see Remark 3.5, but here we discuss some algebraic applications. In the last two sections of this paper we discuss results for derived equivalences of rings and differential graded algebras. The first of these results shows that any derived equivalence of rings lifts to the stronger "up to homotopy" equivalence mentioned above; see Theorem 4.1. As a consequence, all possible homotopy invariants are preserved by derived equivalences of rings, including algebraic $K$-theory; see Corollary 4.2. The next results show that this is not the case for differential graded algebras. Example 4.5 produces two DGAs whose derived categories are equivalent as triangulated categories but which have non-isomorphic $K$-theories. In Section 5 we also give an explicit example of a derived equivalence of differential graded algebras that does not arise from an algebraic tilting complex. Instead there is a topological tilting spectrum which comes from considering DGAs as examples of ring spectra. 
Acknowledgments: This paper is based on a talk I gave at the MSRI conference "Commutative Algebra: Interactions with Homological Algebra and Representation Theory" in February of 2003. I'd like to thank John Greenlees for the invitation to speak at that conference and Henning Krause for the invitation to write these notes. I'd also like to thank Dan Dugger and Stefan Schwede for the enjoyable collaborations which led to this work.

\section{Spectral Algebra}

Before considering "rings up to homotopy" we must consider "abelian groups up to homotopy". The analogue of an abelian group here is a spectrum. Each spectrum corresponds to a generalized cohomology theory. For example, the Eilenberg-Mac Lane spectrum $H A$ is associated with ordinary cohomology with coefficients in the abelian group $A$. Another well-known cohomology theory is complex $K$-theory; $K^{*}(X)$ basically classifies the complex vector bundles on $X$ and the associated spectrum is denoted $K$. The analogue of a ring is then a generalized cohomology theory with a product. Both $H A$ and $K$ are ring spectra; the associated products are the cup product and the product induced by the tensor product of vector bundles.

At a first approximation, a spectrum is a sequence of pointed spaces. Each of these spaces represents one degree of the cohomology theory; for example $H^{n}(X ; A)$, the $n$th ordinary cohomology of a space $X$, is isomorphic to homotopy classes of maps from $X$ with a disjoint base point added to $K(A, n)$, denoted $\left[X_{+}, K(A, n)\right]$. Here $K(A, n)$ is the Eilenberg-Mac Lane space whose homotopy type is determined by having homotopy concentrated in degree $n, \pi_{n} K(A, n)=A$; for example, $K(\mathbb{Z}, 1)$ is the circle $S^{1}$. Complex $K$-theory is represented by the infinite unitary group $U$ in odd degrees and by the classifying space cross the integers, $B U \times \mathbb{Z}$, in even degrees.

One needs additional structure on a sequence of pointed spaces though to make sure the associated homology theory satisfies the Eilenberg-Steenrod axioms for a homology theory. (Note that since we are considering generalized homology theories here we remove the dimension axiom.) To introduce this structure we need the following pointed version of the Cartesian product of spaces. The smash product should be thought of as an analogue of the tensor product in algebra; here the base point acts like a zero element.

Definition 2.1. The smash product $X \wedge Y$ of two pointed spaces $X$ and $Y$ with base points $p t_{X}$ and $p t_{Y}$ is given by $X \wedge Y=X \times Y /\left(X \times p t_{Y}\right) \cup\left(p t_{X} \times Y\right)$. The suspension of a pointed space $X, \Sigma X$ is defined by $S^{1} \wedge X$.

Finally we give the formal definition of a spectrum.

Definition 2.2. A spectrum $\mathrm{Y}$ is a sequence of pointed spaces $\left(Y_{1}, Y_{2}, \cdots, Y_{n}, \cdots\right)$ with structure maps $\Sigma Y_{n} \longrightarrow Y_{n+1}$. A map of spectra $f: Y \longrightarrow Z$ is given by a sequence of maps $f_{n}: Y_{n} \longrightarrow Z_{n}$ which commute with the structure maps.

Given any pointed space $X$, there is an associated suspension spectrum $\Sigma^{\infty} X$ given by $\left(X, \Sigma X, \Sigma^{2} X, \cdots\right)$. A particularly important example is the sphere spectrum, $\mathbb{S}=\Sigma^{\infty} S^{0}$ given by $\left(S^{0}, S^{1}, S^{2}, \cdots\right)$ since the suspension of the $n$-sphere is the $n+1$-sphere. 
We should mention here that in general the association of a cohomology theory to a spectrum $Y$ is not as simple as the formula given for $H A$ and $K$. We explain this point in Remark 3.2.

As mentioned above, a ring spectrum is a spectrum associated to a generalized cohomology theory with a product. Ordinary cohomology theory with coefficients in a ring $R$ has such a product given by the cup product $H^{p}(X ; R) \otimes H^{q}(X ; R) \longrightarrow$ $H^{p+q}(X ; R)$. This product is induced by a compatible family of maps on the associated spaces: $K(R, p) \wedge K(R, q) \longrightarrow K(R, p+q)$. Basically then, a ring spectrum $R$ is a spectrum with compatibly associative and unital products $R_{p} \wedge$ $R_{q} \longrightarrow R_{p+q}$. The unital condition here ensures that these products interact in a compatible way with the suspension structure as well. Unfortunately, although this simple outline is a good beginning for the definition of ring spectra, no one has actually been able to finish it in a way that captures the objects we actually care about.

One wants to define a smash product on the category of spectra which acts like a tensor product, that is, is a symmetric monoidal product. Then the ring spectra would be spectra $R$ with an associative and unital product map $R \wedge R \longrightarrow R$. The problem here is that one must choose a sequence of spaces to define a spectrum $R \wedge R$ from among the two-dimensional array of spaces $R_{p} \wedge R_{q}$. Boardman gave the first approximation to defining such a smash product; his version is only commutative and associative up to homotopy [Bo]; see also [Ad, III]. Using this smash product one can instead consider $A_{\infty}$ or $E_{\infty}$ ring spectra, which are associative or commutative rings up to all higher homotopies [Ma2]. These definitions are cumbersome in comparison to the algebraic analogues though.

In 1991, Gaunce Lewis published a paper which seemed to imply that these definitions were as good as could be [Le]. He showed that no smash product exists which satisfies five reasonable axioms. One of his axioms was that the smash product is strictly commutative and associative; the other axioms asked for reasonable relationships between the smash product for spectra and the smash product for spaces.

Luckily, it turns out that Lewis' axioms were just a bit too strong. In the last few years, several new ways of defining categories of spectra with a good smash product have been discovered. In these categories the smash product acts like a tensor product and the usual algebraic definitions capture the correct notion of ring, algebra and module spectra. This has made it possible to really do algebra in the setting of stable homotopy theory.

Rather than give an overview of the different categories and products that have been defined, we concentrate on the one version, symmetric spectra, which has proved most useful for comparisons with algebra. The definitions for symmetric spectra are also the closest to the simple approximate definitions above. Because the different models for spectra all agree 'up to homotopy', in a way we discuss in Remark 3.4, one can always choose to use whichever category is most convenient to the problem at hand.

Definition 2.3. [HSS] A symmetric sequence is a sequence of pointed spaces, $\left(X_{1}, X_{2}, \cdots\right)$, with an action of $\Sigma_{n}$, the $n$th symmetric group, on $X_{n}$, the $n$th space. For two symmetric sequences $X$ and $Y$, define $X \otimes Y$ as the sequence with $(X \otimes Y)_{n}=\bigvee_{p+q=n} \Sigma_{n} \wedge_{\Sigma_{p} \times \Sigma_{q}}\left(X_{p} \wedge Y_{q}\right)$ 
The suspension spectra introduced above are examples of symmetric sequences with the symmetric action taking place on the suspension coordinates. That is, $\Sigma^{n} X=S^{1} \wedge S^{1} \cdots S^{1} \wedge X$ and the copies of $S^{1}$ are permuted. In particular, the sequence of spheres, $\mathbb{S}$, is a commutative ring under this product; specifically, there is an associative, commutative and unital map $\mathbb{S} \otimes \mathbb{S} \longrightarrow \mathbb{S}$ induced by the $\Sigma_{p} \times \Sigma_{q^{-}}$ equivariant maps $S^{p} \wedge S^{q} \longrightarrow S^{p+q}$. Here we define $S^{n}=\left(S^{1}\right)^{\wedge n}=S^{1} \wedge S^{1} \cdots S^{1}$. Definition 2.4. [HSS] A symmetric spectrum is a module over the commutative ring $\mathbb{S}$ in the category of symmetric sequences. This module structure on a symmetric sequence $X$ is determined by an associative and unital map $\alpha_{X}: \mathbb{S} \otimes X \longrightarrow$ $X$. Unraveling this further, a symmetric spectrum $X$ is a symmetric sequence $\left(X_{1}, X_{2}, \cdots\right)$ with compatible $\Sigma_{p} \times \Sigma_{q}$-equivariant maps $S^{p} \wedge X_{q} \longrightarrow X_{p+q}$. The smash product $X \wedge Y=X \otimes_{\mathbb{S}} Y$ is the coequalizer of the two maps $1 \otimes \alpha_{Y}, \alpha_{X} \otimes 1$ from $X \otimes \mathbb{S} \otimes Y$ to $X \otimes Y$. Similarly, a symmetric ring spectrum is an $\mathbb{S}$-algebra; specifically, a symmetric ring spectrum $R$ is a symmetric sequence with compatible unit $\operatorname{map} \eta: \mathbb{S} \longrightarrow R$ and associative multiplication map $\mu: R \otimes R \longrightarrow R$.

The components of the multiplication map for a symmetric ring spectrum $R$ are $\Sigma_{p} \times \Sigma_{q}$ equivariant maps $R_{p} \wedge R_{q} \longrightarrow R_{p+q}$. For the rest of this article we shorten "symmetric ring spectrum" to just "ring spectrum"; in particular, note that a commutative symmetric ring spectrum would have the added condition that $\mu \tau=\mu$ where $\tau$ is the twist map on $R \wedge R$. For $R$ a (commutative) ring spectrum, the definitions of $R$-modules and $R$-algebras follow similarly.

The Eilenberg-Mac Lane spectrum $H R$ for $R$ any classical ring plays an important role in this paper. Since we want $H R$ to be a ring spectrum (commutative if $R$ is), we need to be careful about our choice of the spaces $K(R, n)$. It is easiest to define $K(R, n)$ as a simplicial set and then take its geometric realization if we want to work with topological spaces; see [GJ, Ma1]. (Actually, throughout this paper "space" can be taken to mean either simplicial set or topological space.) Define the simplicial set $S^{1}$ to be $\Delta[1] / \partial \Delta[1]$, the one-simplex with its two endpoints identified. Then define $H R_{n}=K(R, n)$ to be the simplicial set which in level $k$ is the free $R$-module with basis the non-basepoint $k$-simplices of $S^{n}=\left(S^{1}\right)^{\wedge n}[\mathrm{HSS}$, $1.2 .5]$.

\section{Quillen model CATEGories}

In algebra one considers derived categories; given an abelian category $\mathcal{A}$, the derived category of $\mathcal{A}, \mathcal{D}(\mathcal{A})$, is the localization obtained from the category $\mathcal{C h}(\mathcal{A})$ of (unbounded) chain complexes in $\mathcal{A}$ by inverting the quasi-isomorphisms, or maps which induce isomorphisms in homology. The analogues in homotopy theory are homotopy categories; beginning with a category $\mathcal{C}$ and a notion of weak equivalence the homotopy category $\operatorname{Ho}(\mathcal{C})$ is obtained by inverting the weak equivalences. By requiring more structure on $\mathcal{C}$, namely a Quillen model structure, one can avoid the set theoretic difficulties which exist when inverting a general class of morphisms. This extra structure also enables the application of standard techniques of homotopy theory and captures more homotopical information than the homotopy category alone; see Section 5 for an example of two non-Quillen equivalent model categories with equivalent homotopy categories.

A Quillen model category is a category $\mathcal{C}$ with three distinguished types of maps called weak equivalences, cofibrations and fibrations which satisfy the five axioms below [Q]. A modern variation on these axioms appears in [Ho], and [DwSp] is a 
very good, short introduction to model categories. It may be useful when reading these axioms to keep a couple of archetypal examples in mind. The category of bounded below chain complexes of $R$-modules $\mathcal{C} h_{+}(R)$, is a Quillen model category with weak equivalences the quasi-isomorphisms, cofibrations the injections with levelwise projective cokernels and fibrations the surjections. Similarly, $\mathcal{C} h_{+}(R)$ with the same weak equivalences, cofibrations the injections and fibrations the surjections with levelwise injective kernels is also a Quillen model category. These structures can both be extended to the category of unbounded chain complexes but the cofibrations in the first (projective) case and the fibrations in the second (injective) case are less explicitly defined [Ho]. The homotopy categories associated to these model categories are equal (as triangulated categories) to the corresponding (bounded or unbounded) variants of the derived category of $R$; see [We, 10.3.2].

Axioms 3.1. [Q] Axioms for a Quillen model category.

(1) $\mathcal{C}$ admits all finite limits and colimits.

(2) If two out of three of $f, g$ and $g f$ are weak equivalences, so is the third.

(3) Cofibrations, fibrations and weak equivalences are closed under retract.

(4) Any map $f$ may be factored in two ways: $f=p i$ with $i$ a cofibration and $p$ a fibration and a weak equivalence ( $a$ trivial fibration), and $f=q j$ with $j$ a cofibration and weak equivalence ( $a$ trivial cofibration) and $q$ a fibration.

(5) Given a commuting square

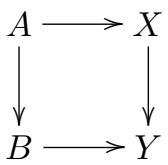

with $A \longrightarrow B$ a cofibration and $X \longrightarrow Y$ a fibration, then a morphism $B \longrightarrow X$ making both triangles commute exists if either of the two vertical maps is a weak equivalence.

Remark 3.2. To give some feel for working with a model category, note that in the projective model category on $\mathcal{C} h_{+}(R)$ the first factorization mentioned in (4) above applied to a map $0 \longrightarrow M$ (with $M$ an $R$-module concentrated in degree zero) produces a projective resolution of $M$. Similarly, the second factorization produces injective resolutions in the injective model category on $\mathcal{C} h_{+}(R)$.

Also, the formula mentioned above for associating a cohomology theory to a spectrum works only for fibrant spectra. An object in a model category is fibrant if the map $Y \longrightarrow *$ is a fibration, where $*$ is the terminal object. In the standard model category structures on both symmetric spectra and the category of spectra in Definition 2.2, it turns out that $Y$ is fibrant if and only if $Y_{n} \longrightarrow \operatorname{map}_{*}\left(S^{1}, Y_{n+1}\right)=$ $\Omega Y_{n+1}$ is a weak equivalence of spaces where this map is adjoint to the map $\Sigma Y_{n} \longrightarrow$ $Y_{n+1}$. Thus, for $Y$ fibrant $\left[X, Y_{n}\right] \cong\left[X, \Omega Y_{n+1}\right] \cong\left[\Sigma X, Y_{n+1}\right]$; this corresponds to the fact that for any cohomology theory the cohomology group in degree $n+1$ of $\Sigma X$ agrees with the $n$th cohomology group of $X$. A weakly equivalent fibrant replacement always exists due to axiom (4); one factors the map $Y \longrightarrow *$ as $Y \longrightarrow$ $Y^{f} \longrightarrow *$ with $Y \longrightarrow Y^{f}$ a weak equivalence and $Y^{f} \longrightarrow *$ a fibration. Then, for a general spectrum $Y$, the associated cohomology theory is given by homotopy classes of maps into the levels of $Y^{f}$.

We also have the following notion of equivalence between model categories. 
Definition 3.3. $[\mathrm{Q}, \mathrm{Ho}]$ A Quillen adjunction between two Quillen model categories $\mathcal{C}$ and $\mathcal{D}$ is given by an adjoint pair of functors $L: \mathcal{C} \rightleftarrows \mathcal{D}: R$ where the left adjoint, $L$, preserves cofibrations and the right adjoint, $R$, preserves fibrations. It follows that $L$ also preserves trivial cofibrations and $R$ preserves trivial fibrations. Under these conditions the adjoint functors induce adjoint derived functors on the homotopy categories $\bar{L}:$ Ho $\mathcal{C} \rightleftarrows \mathrm{Ho} \mathcal{D}: \bar{R}$. This pair is a Quillen equivalence if $\bar{L}$ and $\bar{R}$ form an equivalence of categories between $\operatorname{Ho} \mathcal{C}$ and Ho $\mathcal{D}$. Two model categories are said to be Quillen equivalent if there is a string of Quillen equivalences between them.

We call all of the homotopical information associated to a Quillen model structure a homotopy theory. For example, the identity functors between the projective and injective model categories defined above on $\mathcal{C} h_{+}(R)$ form a Quillen equivalence. That is, the projective and injective model categories define the same homotopy theory, which encompasses the homological algebra associated with $R$.

Remark 3.4. The category of spectra in Definition $2.2[\mathrm{BF}]$, the category of symmetric spectra [HSS] and all of the other new categories of spectra [EKMM, Ly1, Ly2, MMSS] have Quillen equivalent model structures [MMSS, Sch1]. Moreover, the associated categories of rings, modules and algebras are also Quillen equivalent. This is the sense referred to in Section 2 in which these models all agree 'up to homotopy'; since Quillen equivalent model structures define the same homotopy theory, any homotopically invariant statement about one model applies to the others as well.

These models for spectra actually define stable homotopy theories; that is, theories where suspension is invertible in the homotopy category. Suspension corresponds to shifting the spaces in a spectrum down by one; this is easiest to see with suspension spectra. One can show that up to homotopy this functor is invertible with inverse given by shifting up by one. The category $\mathcal{C h}(R)$ is another example of a stable homotopy theory; again suspension and desuspension are homotopic to shifting. The homotopy category associated to a stable homotopy theory is a triangulated category $[\mathrm{Ho}, 7.1 .6]$; the triangles are given by the homotopy fiber sequences (which can be shown to agree with the homotopy cofiber sequences). In particular, Quillen equivalences between stable model categories induce triangulated equivalences on the homotopy categories.

We end this section with two remarks related to Theorem 1.3 and Quillen equivalences.

Remark 3.5. There have been many applications of variations of Theorem 1.3 in stable homotopy theory. A variation on this theorem shows that any stable model category with a compact generator is Quillen equivalent to modules over a ring spectrum [SS2]. This shows that many homotopical settings can be translated into settings in spectral algebra. Applications of this variation include various characterizations of the stable homotopy theory of spectra [SS3, Sh1, Sch2]. Since any rational ring spectrum is an $H \mathbb{Q}$-algebra, combining this theorem with Theorem 4.3 gives algebraic models for any rational stable homotopy theory [Sh3]. This is used in [Sh2, GS] to form practical algebraic models for rational torus-equivariant spectra for tori of any dimension. 
Remark 3.6. In [SS2] condition (1) of Theorem 1.3 actually requires that the functors involved in the Quillen equivalences preserve the enrichment of these module categories over spectra. In [DS3] we show that this requirement is not actually necessary by using ideas similar to those in [Du1, Du2].

\section{Differential graded Algebras}

In the rest of this paper we explore some results which lie between the Morita theory for derived categories of rings and the Morita theory for ring spectra.

First, we consider the overlap between Theorems 1.2 and 1.3. Both of these theorems apply to rings since there is an Eilenberg-Mac Lane ring spectrum associated to any ring. Then we extend the association between spectra and classical algebraic objects to differential graded rings, modules and algebras and their associated homotopy theories. This shows that the category of DGAs lies between rings and ring spectra. Finally, we discuss the Morita theory of DGAs.

Since any Quillen equivalence induces an equivalence on the homotopy (or derived) categories, Theorem 1.3 may seem to give a stronger result for ring spectra than Theorem 1.2 does for rings. Theorem 1.3, however, has a stronger hypothesis on the endomorphism ring of the generator since the weak equivalence type of a ring spectrum is a finer invariant in general than the homotopy ring. (Here the graded ring of homotopy groups of $\overline{\operatorname{Hom}}_{R^{\prime}}(M, M)$ is isomorphic to the graded ring of endomorphisms of $M$ in $\mathrm{Ho}\left(R^{\prime}\right.$-modules $)$.) When that homotopy is concentrated in degree zero though, the weak equivalence type of a ring spectrum is determined by its homotopy. Thus, for an Eilenberg-Mac Lane spectrum the conditions on the generator in the two theorems are equivalent. From this one can show that for rings the notions of Quillen equivalence and derived equivalence actually agree.

Theorem 4.1. [DS1] For two rings $R$ and $R^{\prime}$ the following are equivalent.

(1) The derived categories $\mathcal{D}(R)$ and $\mathcal{D}\left(R^{\prime}\right)$ are triangulated equivalent.

(2) $\mathcal{C h}(R)$ and $\mathcal{C h}\left(R^{\prime}\right)$ are Quillen equivalent model categories.

(3) There is a compact generator $M$ in $\mathcal{D}\left(R^{\prime}\right)$ such that the graded endomorphism ring in the derived category $\mathcal{D}\left(R^{\prime}\right)(M, M)_{*}$ is isomorphic to $R$.

This is one case where the homotopy (or derived) category actually determines the whole homotopy theory. For this reason, one expects a derived equivalence between two rings to induce an isomorphism on any homotopy invariant of rings. For example, Hochschild homology and cyclic homology have been shown to be invariants of derived equivalences [R1, R2, Ke2]; see also [Ke3]. One can also show that a Quillen equivalence preserves algebraic $K$-theory. We have the following result. For regular rings this can also be derived from Neeman's work on the $K$ theory of abelian categories [Ne2, Ne3, Ne4, Ne5, Ne6]; this result also appears in $[\mathrm{DG}]$ with flatness conditions.

Corollary 4.2. [DS1] If $\mathcal{D}(R)$ and $\mathcal{D}\left(R^{\prime}\right)$ are triangulated equivalent, then $K_{*}(R) \cong$ $K_{*}\left(R^{\prime}\right)$.

Just as the Eilenberg-Mac Lane ring spectrum $H R$ represents ordinary cohomology with coefficients in $R$, there is a ring spectrum $H A$ associated to hypercohomology with coefficients in a DGA $A$. Here $H$ is defined as a composite of several functors, so we do not define it explicitly for DGAs. In fact $H$ induces a Quillen equivalence between $H \mathbb{Z}$-algebras and DGAs. The following statement collects the various related results. For the relevant Quillen model structures, see [HSS, SS1]. 
Theorem 4.3. (1) $[\mathrm{SS} 2, \mathrm{Ro}]$ For any classical ring $R$, the model categories of $\mathrm{Ch}(R)$ and $H R$-modules are Quillen equivalent.

(2) [Sh3] The model categories of differential graded algebras and $H \mathbb{Z}$-algebras are Quillen equivalent.

(3) [Sh3] For any DGA A, the model categories of differential graded A-modules and $H A$-modules are Quillen equivalent.

Using this theorem to consider DGAs as lying between rings and ring spectra, one might conjecture that there is an intermediary Morita theory for DGAs similar to Theorem 4.1 for rings. The homology ring, however, does not determine the quasi-isomorphism type for a DGA; for example, a non-formal DGA is not quasiisomorphic to its homology (see Section 5 for an example). Thus, a Morita theorem for DGAs must require a condition on the quasi-isomorphism type of the derived endomorphism DGA of a generator rather than just its homology. (Here the derived endomorphism DGA is an analogue of the derived endomorphism ring spectrum.) This leads to the following question.

Question 4.4. For two DGAs $A$ and $B$, are the following equivalent?

(1) $\mathcal{D}(A)$ is triangulated equivalent to $\mathcal{D}(B)$.

(2) The model categories of differential graded modules over $A$ and $B$ are Quillen equivalent.

(3) There is a compact generator $M$ in $\mathcal{D}(A)$ whose derived endomorphism $D G A$ is quasi-isomorphic to $B$.

If a generator $M$ exists which satisfies (3), then one can show that the model categories of differential graded modules over $A$ and $B$ are Quillen equivalent and hence $\mathcal{D}(A)$ and $\mathcal{D}(B)$ are triangulated equivalent. In fact this follows from Theorems 4.3 and 1.3 by considering the DGAs as examples of ring spectra and replacing differential graded modules by module spectra. Hence, indeed (3) implies (2) implies (1). The other implications fail though. In Example 4.5 below we give an example of a derived equivalence with no underlying Quillen equivalence; so (1) does not imply (2). In Section 5 below we'll also give an example of a Quillen equivalence between DGAs with no generator satisfying (3); so (2) does not imply (3). This example arises from considering DGAs as special examples of ring spectra.

Example 4.5. There are two DGAs $A$ and $B$ whose derived categories are equivalent even though the associated model categories of differential graded modules are not Quillen equivalent. In particular, Corollary 4.2 does not extend to DGAs because $A$ and $B$ have non-isomorphic $K$-theories. This example is based on Marco Schlichting's work in [Schl] which shows that for $p>3$ the stable module categories over $(\mathbb{Z} / p)[\epsilon] / \epsilon^{2}$ and $\mathbb{Z} / p^{2}$ are triangulated equivalent but the associated $K$-theories are not isomorphic.

Proposition 4.6. [DS2] The model category underlying each of these stable module categories is Quillen equivalent to a category of differential graded modules over a $D G A$.

$$
\begin{gathered}
\operatorname{Stmod}\left((\mathbb{Z} / p)[\epsilon] / \epsilon^{2}\right) \simeq \text { Quillen d. g. Mod- } A \\
\operatorname{Stmod}\left(\mathbb{Z} / p^{2}\right) \simeq \text { Quillen d. g. Mod- } B
\end{gathered}
$$

For the dual numbers, the DGA $A$ is $\mathbb{Z} / p\left[x, x^{-1}\right]$, a polynomial algebra on a class in degree one and its inverse with trivial differential. Let $k=\mathbb{Z}\left[x, x^{-1}\right]$, a similarly 
graded polynomial algebra over $\mathbb{Z}$. For $\mathbb{Z} / p^{2}$, the DGA $B$ is generated over $k$ by a class $e$ in degree one with the relations that $e^{2}=0, e x+x e=x^{2}, d e=p$ and $d x=0$. Here $A$ and $B$ are the endomorphism DGAs of the Tate resolution of a generator $(\mathbb{Z} / p)$ of the respective stable module categories [DS2]; see [Ke1, 4.3] for a related general statement.

Because the stable module categories are triangulated equivalent, it follows that $\mathcal{D}(A)$ and $\mathcal{D}(B)$ are also equivalent. Since Quillen equivalences induce isomorphisms in $K$-theory [DS1], Schlichting's work showing the stable module categories' $K$ theories are non-isomorphic implies that the model categories underlying the stable module categories are not Quillen equivalent. Hence the differential graded modules over $A$ and $B$ are not Quillen equivalent, either.

\section{Two topologically EQUivalent DGAs}

As mentioned above, in this section we discuss an example from [DS3] of two DGAs $A$ and $B$ with Quillen equivalent categories of differential graded modules where there is no generator satisfying the properties listed in Question 4.4. This example arises from replacing the DGAs by their associated $H \mathbb{Z}$-algebras $H A$ and $H B$. One can then forget the $H \mathbb{Z}$-algebra structure and consider them as ring spectra, or $\mathbb{S}$-algebras. Since some structure has been forgotten it is reasonable to expect that there are more maps and equivalences between $H A$ and $H B$ as $\mathbb{S}$-algebras than between the DGAs $A$ and $B$. This leads to the following definition.

Definition 5.1. Two DGAs $A$ and $B$ are topologically equivalent if their associated $H \mathbb{Z}$-algebras $H A$ and $H B$ are equivalent as ring spectra ( $\mathbb{S}$-algebras).

Quasi-isomorphic DGAs are topologically equivalent as well, but the converse does not hold. Below we give an example of two DGAs $A$ and $A^{\prime}$ which are topologically equivalent but not quasi-isomorphic. Moreover we show that these two DGAs have equivalent derived categories even though there is no compact generator in $\mathcal{D}\left(A^{\prime}\right)$ whose derived endomorphism DGA is quasi-isomorphic to $A$. In fact, any two topologically equivalent DGAs have equivalent derived categories.

Theorem 5.2. If $A$ and $B$ are topologically equivalent DGAs then $\mathcal{D}(A)$ is triangulated equivalent to $\mathcal{D}(B$.$) . Moreover, the associated categories of differential$ graded modules are Quillen equivalent.

This is based on the following.

Corollary 5.3. If $R \longrightarrow R^{\prime}$ is a weak equivalence of ring spectra, then the model categories of $R$-module spectra and $R^{\prime}$-module spectra are Quillen equivalent.

This follows from Theorem 1.3 by taking $R^{\prime}$ as the compact generator required in condition (2) since $\overline{\operatorname{Hom}}_{R^{\prime}}\left(R^{\prime}, R^{\prime}\right)=R^{\prime}$ is weakly equivalent to $R$. Although this is stated as a corollary here, it is actually an ingredient in the proof of Theorem 1.3.

Proof of Theorem 5.2. By Theorem 4.3 differential graded modules over $A$ and module spectra over $H A$ are Quillen equivalent and the same is true for $B$ and $H B$. Then Corollary 5.3 provides the bridge between these pairs showing that $H A$-module spectra and $H B$-module spectra are Quillen equivalent since $H A$ and $H B$ are weakly equivalent. Since Quillen equivalences between stable model categories induce triangulated equivalences on the homotopy (or derived) categories, the first statement follows from the second. 
Now we introduce the two DGAs from [DS3] which are topologically equivalent but not quasi-isomorphic. The first DGA we consider is a truncated polynomial ring over the integers on a class in degree one, $A=\mathbb{Z}[e] /\left(e^{4}\right)$, with $d(e)=2$. Note that $A$ is not graded commutative, since $e^{2}$ is not trivial. Since $d\left(e^{2}\right)=0$ and $d\left(e^{3}\right)=2 e^{2}$, the homology of $A$ is an exterior algebra over $\mathbb{Z} / 2$ on a class in degree 2. Let $A^{\prime}=H_{*} A=\Lambda_{\mathbb{Z} / 2}\left(\alpha_{2}\right)$, with trivial differential.

One can show that $A$ and $A^{\prime}$ are not quasi-isomorphic. In fact, $A$ is not quasiisomorphic to any DGA over $\mathbb{Z} / 2$. In particular, there are no maps from $\mathbb{Z} / 2$, thought of as a DGA concentrated in degree zero, to $A$ even up to homotopy; that is, $[\mathbb{Z} / 2, A]=0$ in the homotopy category of associative DGAs. On the other hand, any DGA over $\mathbb{Z} / 2$ would have a unit map to it from $\mathbb{Z} / 2$. To calculate that $[\mathbb{Z} / 2, A]=0$, one replaces $\mathbb{Z} / 2$ by a quasi-isomorphic free associative DGA over $\mathbb{Z}$ and shows that there are no maps from it to $A$. In low degrees, one possibility for this replacement begins with a generator $x$ in degree one with $d x=2$, a generator $y$ in degree three with $d y=x^{2}$ and other generators in degree four or higher.

This also shows that there is no compact generator $M$ in $\mathcal{D}\left(A^{\prime}\right)$ whose derived endomorphism DGA is quasi-isomorphic to $A$. Since $A^{\prime}$ is a DGA over $\mathbb{Z} / 2$, all of the homomorphism groups between differential graded $A^{\prime}$ modules are naturally $\mathbb{Z} / 2$ vector spaces. It follows that the derived endomorphism DGA of any differential graded $A^{\prime}$ module would also be a DGA over $\mathbb{Z} / 2$, so this endomorphism DGA cannot be quasi-isomorphic to $A$. Once we show that $A$ and $A^{\prime}$ are topologically equivalent, $A$ and $A^{\prime}$ give a counter example to the equivalence of conditions (2) and (3) in Question 4.4 by Theorem 5.2.

We use topological Hochschild cohomology, $T H H^{*}$, to show that the associated ring spectra $H A$ and $H A^{\prime}$ are weakly equivalent, or $A$ and $A^{\prime}$ are topologically equivalent. This is the analogue of Hochschild cohomology, $H H^{*}$, for spectral algebra. For a ring $R$ and an $R$-bimodule $M$, DGAs with non-zero homology $H_{0}=R$ and $H_{n}=M$ are classified by $H H_{\mathbb{Z}}^{n+2}(R ; M)$. Similarly, ring spectra with non-zero homotopy $\pi_{0}=R$ and $\pi_{n}=M$ are classified by $T H H_{\mathbb{S}}^{n+2}(H R ; H M)$ [La].

For $R$ a $k$-algebra and $M$ an $R$-bimodule over $k, H H_{k}^{*}(R ; M)$, is calculated as the derived bimodule homomorphisms from $R$ to $M$ over the derived tensor product $R \bar{\otimes}_{k} R^{O P}$. In the case where $k$ is a field, it is not necessary to derive the tensor product $R \otimes_{k} R^{O p}$ so this extra wrinkle is often suppressed. As an example, one can calculate that $\mathbb{Z} / 2 \bar{\otimes}_{\mathbb{Z}} \mathbb{Z} / 2$ is quasi-isomorphic to the exterior algebra $\Lambda_{\mathbb{Z} / 2}\left(x_{1}\right)$, so $H H_{\mathbb{Z}}^{*}(\mathbb{Z} / 2 ; \mathbb{Z} / 2)=\operatorname{Ext}_{\Lambda_{\mathbb{Z} / 2}\left(x_{1}\right)}(\mathbb{Z} / 2, \mathbb{Z} / 2)=\mathbb{Z} / 2\left[\sigma_{2}\right]$.

$T H H^{*}$ is defined similarly, by considering maps of bimodule spectra. In particular, $T H H_{H \mathbb{Z}}^{*}(H R ; H M)=H H_{\mathbb{Z}}^{*}(R, M)$, although $T H H^{*}$ can be considered over any ring spectrum. Here we need to consider $T H H_{\mathbb{S}}^{*}(H \mathbb{Z} / 2, H \mathbb{Z} / 2)$; by [FLS], this is $\Gamma_{\mathbb{Z} / 2}\left[\tau_{2}\right]$, a divided power algebra over $\mathbb{Z} / 2$ which is isomorphic to an exterior algebra over $\mathbb{Z} / 2$ on classes $e_{i}$ for $i \geq 1$ with $e_{i}$ in degree $2^{i}$.

The unit map $\mathbb{S} \longrightarrow H \mathbb{Z}$ induces a map

$$
\Phi: H H_{\mathbb{Z}}^{*}(\mathbb{Z} / 2, \mathbb{Z} / 2) \longrightarrow T H H_{\mathbb{S}}^{*}(H \mathbb{Z} / 2, H \mathbb{Z} / 2)
$$

The two elements in $H H^{2}$ classify the two rings $\mathbb{Z} / 4$ and $\mathbb{Z} / 2 \oplus \mathbb{Z} / 2$. Since the associated Eilenberg-Mac Lane ring spectra are also distinct, $\Phi$ must be injective in degree two; hence $\Phi(\sigma)=\tau$. Since $A$ and $A^{\prime}$ are not quasi-isomorphic, one can check that $\sigma^{2}$ and 0 in $H H^{4}$ correspond respectively to $A$ and $A^{\prime}$. Since $\tau^{2}=0$ and $\Phi$ is a ring homomorphism, $\Phi\left(\sigma^{2}\right)=0$. So $H A$ and $H A^{\prime}$ correspond to the same 
homotopy type as ring spectra; that is, $H A$ and $H A^{\prime}$ are weakly equivalent and $A$ and $A^{\prime}$ are topologically equivalent.

\section{REFERENCES}

[Ad] Adams, J. Frank, Stable homotopy and generalised homology, University of Chicago Press, Chicago, 1974.

[Bo] Boardman, J.M., Stable homotopy theory, preprint, Warwick University, 1964.

[BN] M. Bökstedt and A. Neeman, Homotopy limits in triangulated categories Compositio Math. 86 (1993), 209-234.

[BF] A. K. Bousfield and E. M. Friedlander, Homotopy theory of $\Gamma$-spaces, spectra, and bisimplicial sets. Geometric applications of homotopy theory (Proc. Conf., Evanston, Ill., 1977), II, pp. 80-130, Lecture Notes in Math., 658, Springer, Berlin, 1978.

[DG] J. Daplayrat-Glutron, Equivalence dérivées et K-théorie (d'après ThomasonTrobaugh), Mémoire de DEA, Université Denis Diderot - Paris7, 1999, http://www.math.jussieu.fr/ keller/

[Du1] D. Dugger, Universal homotopy theories, Adv. Math. 164 (2001), no. 1, 144-176.

[Du2] D. Dugger, Replacing model categories with simplicial ones, Trans. Amer. Math. Soc., 353 (2001), no. 12, 5003-5027.

[DS1] D. Dugger and B. Shipley, K-theory and derived equivalences, Duke J. Math., to appear.

[DS2] D. Dugger and B. Shipley, Stable module categories and differential graded rings, in preparation.

[DS3] D. Dugger and B. Shipley, Topological equivalences of DGAs, in preparation.

[DwSp] W. G. Dwyer and J. Spalinski, Homotopy theories and model categories, Handbook of algebraic topology (Amsterdam), North-Holland, Amsterdam, 1995, pp. 73-126.

[EKMM] A. D. Elmendorf, I. Kriz, M. A. Mandell, and J. P. May, Rings, modules, and algebras in stable homotopy theory. With an appendix by M. Cole, Mathematical Surveys and Monographs, 47, American Mathematical Society, Providence, RI, 1997.

[FLS] Vincent Franjou, Jean Lannes, and Lionel Schwartz, Autour de la cohomologie de Mac Lane des corps finis Invent. Math. 115 (1994), no. 3, 513-538.

[GJ] Paul G. Goerss and John F. Jardine, Simplicial homotopy theory. Progress in Mathematics, 174. Birkhauser Verlag, Basel, 1999.

[GS] J. P. C. Greenlees and B. Shipley, Algebraic models for rational torus-equivariant cohomology theories, in preparation.

[Ho] M. Hovey, Model categories, Mathematical Surveys and Monographs, 63, American Mathematical Society, Providence, RI, 1999.

[HSS] M. Hovey, B. Shipley, and J. Smith, Symmetric spectra, J. Amer. Math. Soc. 13 (2000), 149-208.

[Ke1] B. Keller, Deriving DG categories, Ann. Sci. École Norm. Sup. (4) 27 (1994), 63-102.

$[\mathrm{Ke} 2]$ B. Keller, Invariance and localization for cyclic homology of DG algebras, J. Pure Appl. Algebra 123 (1998), 223-273.

[Ke3] B. Keller, Hochschild cohomology and derived Picard groups, J. Pure Appl. Algebra, to appear.

[La] A. Lazarev, Homotopy Theory of $A_{\infty}$ Ring Spectra and Applications to MU-Modules, K-Theory 24 (3) (2001), 243-281.

[Le] Lewis, L.G., Jr., Is there a convenient category of spectra?, J. Pure and Appl. Alg. 73 (1991), 233-246.

[Ly1] M. Lydakis, Smash products and $\Gamma$-spaces, Math. Proc. Cambridge Philos. Soc. 126 (1999), $311-328$.

[Ly2] M. Lydakis, Simplicial functors and stable homotopy theory, Preprint, Universität Bielefeld, 1998 .

[MMSS] M. A. Mandell, J. P. May, S. Schwede and B. Shipley, Model categories of diagram spectra, Proc. London Math. Soc., 82 (2001), 441-512.

[Ma1] J. P. May, Simplicial Objects in Algebraic Topology, Van Nostrand Mathematical Studies, no. 11, Van Nostrand, Princeton, NJ, 1967.

[Ma2] J. P. May, $E_{\infty}$ ring spaces and $E_{\infty}$ ring spectra. With contributions by Frank Quinn, Nigel Ray, and Jorgen Tornehave. Lecture Notes in Mathematics, 577 Springer-Verlag, Berlin-New York, 1977. 268 pp. 
[Ne1] A. Neeman, The connection between the K-theory localisation theorem of Thomason, Trobaugh and Yao, and the smashing subcategories of Bousfield and Ravenel, Ann. Sci. Ec. Norm. Sup. 25 (1992), 547-566.

[Ne2] A. Neeman, K-theory for triangulated categories I(A): Homological functors, Asian J. Math. Vol 1, no. 2 (1997), 330-417.

[Ne3] A. Neeman, K-theory for triangulated categories. I(B). Homological functors. Asian J. Math. 1 (1997), no. 3, 435-529.

[Ne4] A. Neeman, K-theory for triangulated categories. II. The subtlety of the theory and potential pitfalls. Asian J. Math. 2 (1998), no. 1, 1-125.

[Ne5] A. Neeman, $K$-theory for triangulated categories. III(A). The theorem of the heart. Asian J. Math. 2 (1998), no. 3, 495-589.

[Ne6] A. Neeman, $K$-theory for triangulated categories. III(B). The theorem of the heart. Asian J. Math. 3 (1999), no. 3, 557-608.

[Q] D. G. Quillen, Homotopical algebra, Lecture Notes in Mathematics, 43, Springer-Verlag, 1967.

[R1] J. Rickard, Morita theory for derived categories, J. London Math. Soc. (2) 39 (1989), $436-456$

[R2] J. Rickard, Derived equivalences as derived functors, J. London Math. Soc. (2) 43 (1991), $37-48$.

[Ro] A. Robinson, The extraordinary derived category, Math. Z. 196 (1987), no.2, 231-238.

[Sch1] S. Schwede, S-modules and symmetric spectra, Math. Ann. 319 (2001), 517-532.

[Sch2] S. Schwede, The stable homotopy category has a unique model at the prime 2, Advances in Mathematics 164 (2001), 24-40.

[Sch3] S. Schwede, Morita theory in abelian, derived and stable model categories, Preprint 2003.

[SS1] S. Schwede and B. Shipley, Algebras and modules in monoidal model categories, Proc. London Math. Soc. 80 (2000), 491-511.

[SS2] S. Schwede and B. Shipley, Stable model categories are categories of modules, Topology 42 (2003), 103-153.

[SS3] S. Schwede and B. Shipley, A uniqueness theorem for stable homotopy theory, Mathematische Zeitschrift 239 (2002), 803-828.

[Schl] M. Schlichting, A note on K-theory and triangulated categories, Invent. Math. 150 (2002), no. $1,111-116$.

[Sh1] B. Shipley, Monoidal uniqueness of stable homotopy theory, Advances in Mathematics 160 (2001), 217-240.

[Sh2] B. Shipley, An algebraic model for rational $S^{1}$-equivariant homotopy theory, Quart. J. Math 53 (2002), 87-110.

[Sh3] B. Shipley, HZ-algebra spectra are differential graded algebras, Preprint 2002.

[We] Charles A. Weibel, An introduction to homological algebra. Cambridge Studies in Advanced Mathematics, 38. Cambridge University Press, Cambridge, 1994.

Department of Mathematics, University of Illinois at Chicago, Chicago, IL, USA

E-mail address: bshipley@math.uic.edu 